\title{
Resultados docentes en historia del arte. un camino hacia la motivación y el emprendimiento
}

Teaching results in Art History. A path to motivation and entrepreneurship

\author{
Reyes Escalera Pérez1, Sonia Ríos Moyano2 \\ ${ }^{1}$ Departamento de $\mathrm{H}^{\mathrm{a}}$ del Arte, Universidad de Málaga, España (drescalera@uma.es) \\ ${ }^{2}$ Departamento de $\mathrm{H}^{\mathrm{a}}$ del Arte, Universidad de Málaga, España (srios@uma.es)
}

\section{De enseñar contenidos...}

El aprendizaje en sí es un proceso complejo, y aunque no lo parezca, es una de las bases fundamentales que ha colisionado con las metodologías que tradicionalmente se venían empleando en la Educación Superior. La trasformación tecnológica vivida en las últimas décadas, incomparable con otras innovaciones y avances surgidos en el siglo XX, ha cambiado nuestra forma de comunicarnos, relacionarnos, y sobre todo, aprender. El cerebro humano sigue adaptándose a los cambios y a las herramientas, y éstas, más tecnológicas que nunca, han venido para quedarse, transformar y revolucionar nuestra cotidianeidad hasta límites aún desconocidos.

La adquisición de un nuevo conocimiento, el reconocimiento de una habilidad y el entrenamiento de una capacidad, es una de las claves en el proceso de enseñanza-aprendizaje en el día a día de nuestra docencia, de modo que no es suficiente la traslación y transmisión de contenidos, como si fuera una visión romántica y poética de la historia del arte. No, eso ya no es suficiente. El entrenamiento de la memoria a corto plazo, esa retención pasajera de contenidos, ya pasó a la historia. Ahora nuestra docencia se centra en objetivos y la forma de llevarlos a la práctica, en identificar "problemas" e intentar darles soluciones, en trasladar al aula experiencias que no hay que esperar a tener el título para poder llevarlas a la práctica. Es momento de imaginar, de soñar, de ser creativos, de creer que se puede hacer, es momento de motivar, de enseñarles a emprender, es momento de HACER.

Debemos tener en cuenta los cuatro pilares que cita Jacques Delors en el Informe sobre educación presentado a la UNESCO en 1996: “Aprender a conocer”, “Aprender a hacer”, “Aprender a vivir juntos” y “Aprender a ser” (Delors, 1996, p. 34). “Aprender” implica, además, la recuperación de conocimientos y la reconstrucción de un panorama al que sumamos destrezas y 
actitudes. Si entendemos el aprendizaje desde este punto de vista, no sólo como el vínculo entre estímulo y respuesta, estaremos dando paso a otros modos de aprendizaje que se basan en la imitación, en el descubrimiento autónomo, en la identificación de problemas y la búsqueda de caminos hacia sus soluciones posibles que después transferimos a otras situaciones.

\section{...a crear actividades innovadoras.}

Hay que reconocer que la realización de actividades al cobijo de proyectos de Innovación Educativa es una de las acciones más gratificantes que se realizan durante cada curso académico, puesto que permite la formación y la puesta en práctica de tareas que a priori no se llevarían a cabo, tanto por falta de formación del docente como por falta de respaldo institucional. De tal modo, hay que agradecer a la Universidad de Málaga, gracias a los Servicios encargados de su gestión y a sus responsables, que desde hace años vienen trabajando en la institucionalización de los proyectos de Innovación Educativa. Y lo que en principio surgió como una necesidad ante el cambio que se avecinaba con la entrada de los nuevos grados, se fue convirtiendo en un camino de mejora hacia la calidad docente.

Es por tal motivo que, desde estas líneas, agradezcamos la concesión de los siguientes proyectos de innovación educativa en las convocatorias 2006, 2007, 2013 y 2015 -PIE06/060, PIE07/004, PIE13145 y PIE15-0601-; gracias al trabajo realizado a lo largo de los años, cada vez sumamos nuevos proyectos y experiencias docentes concretadas en actividades motivadoras para los alumnos, las cuales llevan al diseño de formatos de aprendizaje para la experimentación y el ensayo de competencias que exceden los límites de un aula.

Son múltiples y variados los resultados de los dos últimos proyectos, sobre todo, desde los últimos cuatro cursos académicos, en los que profesores que imparten docencia en el Grado de Historia del Arte han aunado esfuerzos para dejar constancia de sus trabajos en el aula2. Entre ellos se encuentran los siguientes: "El arte en el cine y su uso como ampliación del conocimiento del hecho artístico" (Ríos y Escalera, 2014), "Propuestas y reflexiones sobre los modelos de aprendizaje

\footnotetext{
${ }^{1}$ Proyectos de Innovación Educativa, Universidad de Málaga. Coordinados por Sonia Ríos Moyano. Títulos: “Adaptación de asignaturas de Historia del Arte al EEES. Diseño de material didáctico y uso de herramientas telemáticas en el proceso enseñanza-aprendizaje. Experiencia piloto de adaptación al crédito europeo" (Código: PIE06/060); "Historia del Arte hacia el EEES. Desarrollo y experimentación de nuevos modelos didácticos en el proceso enseñanza-aprendizaje. Búsqueda de un espacio tecnológico común para docentes y alumnos” (Código: PIE07/004); “Adecuación de contenidos y actividades formativas para la adquisición de competencias en asignaturas del área de Historia del Arte. Realización de un catálogo de actividades que fomenten la adquisición de competencias y su evaluación". (Código: PIE13-145); "Adecuación de contenidos y actividades formativas para el emprendimiento en artes y humanidades. Adquisición de competencias profesionales y su evaluación” (Código: PIE15-060).

${ }^{2}$ PIE13-145: Coordinadora: Sonia Ríos Moyano. Investigadores: Eugenio Carmona Mato, José Miguel, Morales Folguera, Reyes Escalera Pérez, Rafael Sánchez-Lafuente Gémar, Javier Ordóñez Vergara, Nuria Rodríguez Ortega. PIE15-060: Coordinadora: Sonia Ríos Moyano. Investigadores: Eugenio Carmona Mato, Reyes Escalera Pérez, Rafael Sánchez-Lafuente Gémar y María Josefa de la Torre Molina, Juan Mª Montijano García y José Luis Crespo Fajardo,
} 
en la Historia del arte" (Ríos y Escalera, 2014 y 2017), "Iconografías de la muerte: percepciones y expresiones en la cultura actual. De la teoría al emprendimiento" (Escalera y Ríos, 2016) o “Adaptación de contenidos profesionales a la cultura emprendedora en Historia del Arte" (Escalera y Ríos, 2016).

\section{Gracias a la práctica real, el talento crea conocimiento.}

Por todo ello, en el curso 2014-2015 decidimos organizar un Seminario de ampliación de conocimientos del Grado en Historia del Arte (Universidad de Málaga) que debía vincularse por su temática a las asignaturas en las que impartíamos clase, formando parte de las Actividades formativas de las mismas3.

En primer lugar, entendimos que, tras barajar varias opciones, era la Cultura visual el argumento que debía cohesionar las ponencias que iban a presentarse, siendo ésta una línea de investigación prioritaria en el Departamento de Historia del Arte. En segundo lugar, debíamos decidir a quiénes invitábamos como ponentes; finalmente decidimos dar la oportunidad a graduados y doctorandos que habían realizado sus trabajos Fin de Grado o Fin de Máster sobre la imagen y la retórica verbo-visual bajo nuestra coordinación.

Se tituló "I Seminario de estudios de Cultura Visual” y se celebró en la Facultad de Filosofía y Letras de Málaga el día 20 de noviembre de 2014. Se presentaron diez ponencias, divididas en dos sesiones de mañana y tarde; tras sus exposiciones, se debatió acerca de los asuntos planteados [1].

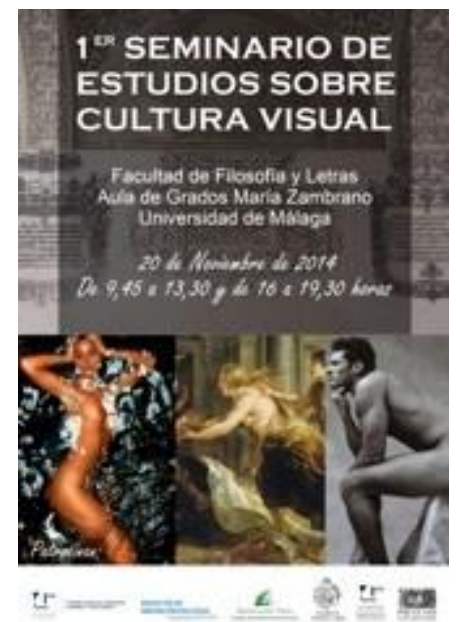

[Fig. 1]: Cartel del "I Seminario de estudios de Cultura Visual”. Sonia Ríos, 2014

\footnotetext{
${ }^{3}$ Las asignaturas son: "Lenguajes artísticos y lecturas de la imagen" (obligatoria, 1er curso), "Diseño y estética de lo cotidiano (obligatoria, 3er curso), "Iconología e iconografía" (optativa, $4^{\circ}$ curso) e "Industria editorial” (optativa, Máster en Desarrollos Sociales de la Cultura Artística).
} 
Éstos fueron los autores y títulos de las ponencias presentadas: María Martínez Yuste: "La influencia de Hendrick Goltzius en los apostolados de la provincia de Málaga"; Lucía Mireya Jiménez Benítez: 'Los tipos iconográficos de 'La tentación' y 'La caída del hombre' en las miniaturas de la Bibliothèque Nationale de Francia"; Miriam Rengel Medina: "La serie de los siete pecados capitales de Brueghel el Viejo. Iconografía"; Sonia Enamorado Corpas: "La moda a través de sus anuncios publicitarios y su relación con la Historia del Arte"; Silvia Cazalla Canto: "La Vanitas como tipo iconográfico en la literatura emblemática hispánica"; Ma Gracia Ortega Martín: "La Tarasca en Granada: símbolo y regocijo (1883-1936)"; José Antonio Vázquez Sánchez: "La Pecadora santa. Pinturas murales del convento de la Magdalena de Antequera: un sermón plástico"; Ana Solís Díaz: "Diagnóstico gráfico del rótulo comercial malagueño (zona centro). Siglo XX y principios del siglo XXI"; Luis Delgado Mata: "El grito de Carmen. Aproximación a las Damas de Saura" y Franziska Koch: "Antropofagia. Mitos clásicos. Arte".

Los ponentes realizaron un resumen de cada una de sus presentaciones que fueron subidas a la plataforma Campus Virtual para que formaran parte del material docente de cada una de las asignaturas vinculadas al proyecto. A todos los asistentes se les entregó la correspondiente certificación de asistencia.

Asimismo nos planteamos que, dada la excelente calidad de los trabajos presentados, debían ser publicados; así también lo entendieron los responsables del Grupo de investigación Eudmed.net (SEJ 309) de la Universidad de Málaga. Bajo su auspicio, se editó en 2015 un libro en su colección "Biblioteca de Ciencias y Artes" con el título: Cultura simbólica. Estudios, en el que se incluyen todas las investigaciones presentadas; además, cuenta con la "Presentación" de otro de los investigadores del PIE, el profesor Rafael Sánchez-Lafuente Gémar.

Dada la buena acogida, decidimos organizar en el curso 2015-2016 el "II Seminario de estudios de Cultura Visual”; en esta ocasión se celebró en la Facultad de Medicina el 26 de noviembre de 2015. Nuevamente fueron diez las ponencias presentadas: Leticia Crespillo Mari: "Itinerarium mentis ad Deum. La luz en los edificios medievales cristianos e islámicos: un análisis comparativo"; María Terrón: "Arte Outsider"; Sandra Medina Bueno: "Pervivencias y trasposiciones de la estética surrealista en el diseño y otras manifestaciones artísticas"; Carmen Rocamora Jiménez: "Más allá de lo efímero. Cinco catafalcos conservados en España y México"; Luis Melero Mascareñas: "La serie de la vida de la Virgen de Juan Correa y 'El Mudo Arellano’ en el Museo de 
la Ciudad de Antequera"; Irene Lara Cuevas: “'Joyas pintadas'. "La platería de oro en los retratos de Antonio Moro, Sánchez Coello y Pantoja de la Cruz"; Marina Caro Rojas: "Instrumentos musicales en la pintura andaluza de los siglos XVII y XVIII”; Macarena López López: “Del 'maricón' al homosexual. Estereotipos en el cine"; Cristina García Pérez: "Creaciones alternativas: los doodles de Google. Recorrido estético a través de un diseño mutante en el año 2014 en España” y Lucía Mireya Jiménez Benítez: "Estudio iconográfico de los vidrios dorados de la Antigua Roma".

Siete de estos trabajos fueron publicados en un nuevo libro titulado Cultura simbólica II. Estudios (2017) con una nueva "Presentación” del doctor Sánchez-Lafuente [2].

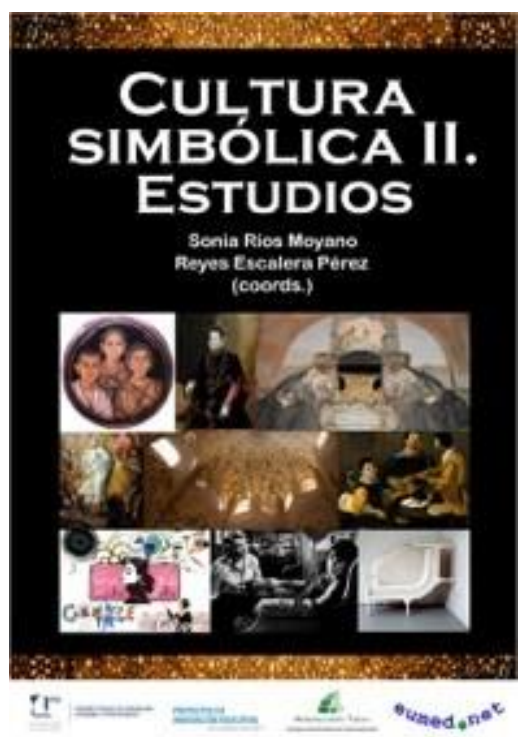

[Fig. 2]: Portada del Libro Cultura simbólica II. Estudios. 2017

Los textos que en esta revista se publican son fruto del "III Seminario de estudios de Cultura Visual" [3] que se celebró en la Facultad de Derecho el 1 de diciembre de 2016. Los trabajos presentados fueron: José Antonio Colón: "La orina, su iconografía y uso en la Historia del Arte"; Gracia Ortega Martín: "La Tarasca. El cambio de identidad y significado en la ciudad de Granada"; Juan José Castillo Herrera: “La 'Apoteosis de Hércules': un discurso humanista en la Sevilla del Renacimiento"; José Antonio Vázquez Sánchez: "Pinturas murales en el convento de la Magdalena en Antequera"; Francisco Jesús Flores Matute: "El patrimonio musical de las hermandades malagueñas. Relación histórica y desarrollo estético de la música"; Leticia Crespillo Marí: "Hacia la configuración de un espacio lumínico desmaterializado. Dos supuestos distantes en el tiempo, una misma aplicación práctica"; Viktoriya Kondratenko Mozgova: "VKHUTEMAS y su huella en el diseño post revolucionario soviético"; Cristina M ${ }^{\mathrm{a}}$ Sánchez Torreblanca: "Estética de la fotografía 
publicitaria"; Sandra Medina Bueno: "La utilización de la Realidad Aumentada en diversos ámbitos de la Historia del Arte” y Catalina Vásquez Hulsbus: "De sierpes, serpientes, culebras y culebrones: propuesta para un estudio iconográfico de los Pliegos de Lira Popular chilena”.

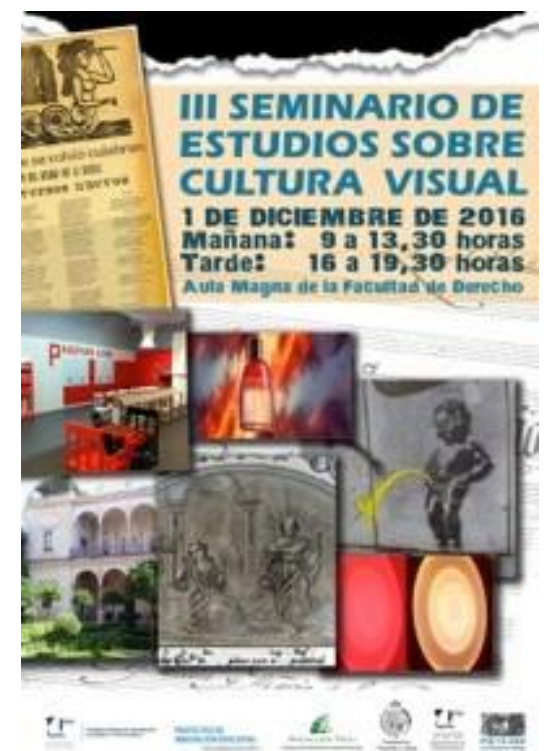

[Fig. 3]: Cartel del "I Seminario de estudios de Cultura Visual". Sonia Ríos, 2017.

Estamos convencidas de que esta actividad ha sido muy enriquecedora para los estudiantes asistentes, y así lo corroboran las encuestas. El primer motivo se relaciona con la obligatoriedad de la realización de un Trabajo Fin de Grado al que se tienen que enfrentar para la obtención del título de graduado. Para muchos alumnos -sobre todo los que cursan primero- esta tarea puede plantearles un duro reto al que temen enfrentarse. Estamos convencidas de que el hecho de ver y escuchar a compañeros que hace sólo unos años tenían su misma incertidumbre les anima y fortalece para desafiar sus probables miedos. Asimismo, les abre un abanico de posibilidades para la decisión de escoger el tema de su trabajo, ya que los que se presentan abarcan numerosos ámbitos y temáticas, algunos apegados a la tradición y otros muchos que rastrean la pervivencia de la retórica en el mundo contemporáneo. Finalmente, creemos que también puede ser un aliciente para ellos abrigar la idea de que en un futuro inmediato puedan ser los ponentes.

Por otra parte, para los egresados que han participado (incluso algunos de ellos han repetido), estos Seminarios suponen un primer contacto no sólo con la investigación sino con la difícil tarea de hablar frente a una nutrida audiencia y en un espacio que por su tamaño y solemnidad puede impresionar -todos se han organizado en los Salones de Grado o Aulas Magnas de las Facultades-. 
Asimismo, la publicación de sus trabajos supone para ellos un interesante aliciente y una recompensa tras las arduas tareas a las que se han tenido que enfrentar.

Por último, para nosotras es un estímulo comprobar que los alumnos asistentes se interesan por las conferencias, participando activamente en los debates que se plantean; asimismo, nos congratula comprobar el entusiasmo con que los ponentes preparan sus exposiciones y cómo el nerviosismo inicial se transforma en satisfacción cuando han finalizado. Por otro lado, es muy satisfactorio que un nuevo proyecto editorial -Eviterna. Revista Universitaria de Humanidades, Arte y Cultura- haya sido concebido por tres graduadas, Leticia Crespillo, $\mathrm{M}^{\mathrm{a}}$ Catalina Vásquez (directoras) y Sandra Medina (editora) que participaron en el II y III Seminario, y que han dado la oportunidad a sus compañeros para publicar sus trabajos en este monográfico que sin duda es el comienzo de un vínculo más, la continuidad de una alianza entre los Seminarios de Cultura Visual y la publicación de las ponencias presentadas como número extraordinario en la revista, a la que auguramos un futuro muy halagüeño.

\section{Referencias bibliográficas}

ESCALERA PÉREZ, Reyes y RÍOS MOYANO, Sonia (2016), “Adaptación de contenidos profesionales a la cultura emprendedora en Historia del Arte", en RODRÍGUEZ TERCEÑO, José (ed.), Libro de actas del Congreso CUICIID 2016, p. 168, Fórum Internacional de comunicación y Relaciones Públicas, Madrid.

ESCALERA PÉREZ, Reyes y RÍOS MOYANO, Sonia (2016), "Iconografías de la muerte: percepciones y expresiones en la cultura actual. De la teoría al emprendimiento", en Experiencias y manifestaciones culturales de vanguardia, Colección McGraw-Hill Education (en prensa).

DELORS, Jacques (coord.) (1996), La educación encierra un tesoro. Informe a la UNESCO de la Comisión Internacional para la Educación del siglo XXI, Ediciones UNESCO, Santillana, París. http://www.unesco.org/education/pdf/DELORS_S.PDF (Consultado: 26/05/2017).

RÍOS MOYANO, Sonia y ESCALERA PÉREZ, Reyes (2014), "El arte en el cine y su uso como ampliación del conocimiento del hecho artístico", El Futuro del Pasado, no 5, pp. 65-89. DOI: http://dx. doi.org/10.14516/fdp.2014.005.001.003. 
RÍOS MOYANO, Sonia y ESCALERA PÉREZ, Reyes (2014), "Propuestas y reflexiones sobre los modelos de aprendizaje en historia del arte", en PÉREZ ACUÑA, Beatriz (coord.), Vectores de la Pedagogía docente actual, pp. 379-407, Visión Libros, Madrid.

RÍOS MOYANO, Sonia y ESCALERA PÉREZ, Reyes (2017), "Propostas e reflexoes sobre os modelos de aprendizagem na História da arte", en PEÑA ACUÑA, Beatriz (coord.), Vetores da pedagodia docente atual, pp. 293-312, Media XXI, Porto. 